\title{
Risk Factors for Tuberculosis in Patients Receiving Tumour Necrosis Factor-a Blockers: What Do We Really Know?
}

\author{
${\text { Philip R. Botha }{ }^{a} \text { Coenraad F.N. Koegelenberg }}^{b}$ \\ Divisions of anfectious Diseases and ${ }^{b}$ Pulmonology, Department of Medicine, Stellenbosch University and \\ Tygerberg Academic Hospital, Cape Town, South Africa
}

Tuberculosis (TB) remains one of the most significant infectious diseases to afflict mankind with an estimated one third of the global population affected by latent TB infection (LTBI) [1]. Controlling the spread of TB is hampered by the global HIV pandemic as well as the inexorable global rise in drug-resistant TB with an estimated 650,000 drug-resistant TB cases diagnosed thus far [2]. It is against this backdrop that tumour necrosis factor- $\alpha$ (TNF- $\alpha$ ) blockers have been introduced to provide new hope to patients with a range of autoimmune inflammatory conditions who failed conventional immunosuppressive therapies.

TNF- $\alpha$ plays a pivotal role in the host immune response against $\mathrm{TB}$, and it has long been predicted that the use of biologic agents blocking this cytokine will increase the risk of TB. In a murine model of LTBI, mice treated with anti-TNF- $\alpha$ antibodies displayed delayed granuloma formation as well as disintegration of existing granulomas with both processes leading to mycobacterial dissemination [3].

TNF- $\alpha$ blockers are associated with a relatively increased risk of TB in the order of 1.6-25 [4]. The wide variation in risk is explained by a number of factors including the type of TNF- $\alpha$ blocker used, the duration of follow-up, the prevalence of TB in the general population, the presence of co-morbid illnesses as well as the type of
(C) 2015 S. Karger AG, Basel

0025-7931/15/0903-0185\$39.50/0 other immunosuppressive therapy used. All TNF- $\alpha$ blockers are associated with an increased risk with adalimumab and infliximab having a 3-4 times increased risk compared to etanercept. Moreover, the clinical presentation of TB in patients receiving TNF- $\alpha$ blockers is often atypical and frequently manifests as extrapulmonary disease [5].

In this issue of Respiration, Borekci et al. [6] report their retrospective analysis of patients who were treated with a TNF- $\alpha$ blocker and followed up for at least 6 months during 2003-2013 at a single centre in Turkey. The most common underlying autoimmune inflammatory conditions were ankylosing spondylitis, rheumatoid arthritis and Behçet's disease. All patients were screened for LTBI by a respiratory physician with a clinical assessment, chest radiograph and tuberculin skin test (TST). Isoniazid (INH) prophylaxis was given for a period of 9 months in cases with a positive TST $\geq 5 \mathrm{~mm}$, in patients with radiological evidence of previous $\mathrm{TB}$ with no history of anti-TB treatment, in cases with close contact with an infectious case in the preceding year or at the discretion of the attending respiratory physician. INH was given to $63.6 \%$ of the cases, with a positive TST being the most common indication. In total, 16 patients were subsequently diagnosed with active $\mathrm{TB}$, which corresponds to an incidence of 466/100,000 population, approximately 22 times higher than the national incidence in Turkey. In
Coenraad F.N. Koegelenberg

Division of Pulmonology, Department of Medicine, Stellenbosch University and Tygerberg Academic Hospital, PO Box 241

Cape Town 8000 (South Africa)

E-Mail coeniefn@sun.ac.za 
keeping with previous reports, extrapulmonary TB was common $(69 \%)$ and half of the cases occurred in patients who received INH prophylaxis.

Interestingly, there was a significant association between Behçet's disease and the risk of TB, and patients who had a strongly positive TST ( $\geq 10 \mathrm{~mm}$ ) paradoxically had a significantly lower risk of developing TB. No significant association between age, gender, smoking history, INH prophylaxis, type of TNF- $\alpha$ blocker and use of other immunosuppressive drugs and risk of TB was identified.

A number of unexplained observations and unanswered questions remain. Firstly, this is the first report identifying Behçet's disease as a risk factor for TB in this population, suggesting that the immunological aberrations implicated in this vasculitis disorder predispose patients to TB more so than other autoimmune/inflammatory conditions.

Secondly, the majority of patients $(>80 \%)$ who developed TB did so more than 1 year after receiving treatment with a TNF- $\alpha$ blocker. It can be inferred that the majority of cases with active TB completed their 9 months' INH prophylaxis prior to the diagnosis of TB. Although INH prophylaxis is effective in preventing active TB in patients with LTBI, the benefit seems to be limited for the duration of the prophylaxis. This raises the question of whether 9 months of INH prophylaxis are adequate in patients who receive ongoing TNF- $\alpha$ blocker therapy beyond this time period. In HIV-infected people, prolonged INH prophylaxis for 36 months has been shown to be superior to 6 months' prophylaxis in preventing TB [7]. Clearly, future studies are indicated to define the optimal duration of prophylaxis in patients treated with TNF- $\alpha$ blockers.

Lastly, the paradoxical protection offered by a strongly positive TST suggests that some patients had a relatively intact protective cellular immune response that was less affected by TNF- $\alpha$ blockage.

The findings of Borekci et al. pose some interesting questions and expose numerous gaps in our knowledge around the use of TNF- $\alpha$ blockers in TB endemic areas. Clearly, further research is needed to best define who constitutes a high-risk patient and which is the optimal regimen for TB prophylaxis in those receiving these immune-modulating drugs.

\section{References}

$\checkmark 1$ Corbett EL, Watt CJ, Walker N, et al: The growing burden of tuberculosis: global trends and interactions with the HIV epidemic. Arch Intern Med 2003;163:1009-1021.

2 World Health Organisation: WHO Global Tuberculosis Report 2014. http://www.who. int/tb/publications/global_report/en (accessed June 17, 2015).

3 Mohan VP, Scanga CA, Yu K, et al: Effects of tumor necrosis factor alpha on host immune response in chronic persistent tuberculosis: possible role for limiting pathology. Infect Immun 2001;69:1847-1855.
4 Solovic I, Sester M, Gomez-Reino JJ, et al: The risk of tuberculosis related to tumour necrosis factor antagonist therapies: a TBNET consensus statement. Eur Respir J 2010;36:11851206.

5 Mohan AK, Coté TR, Block JA, Manadan AM, Siegel JN, Braun MM: Tuberculosis following the use of etanercept, a tumor necrosis factor inhibitor. Clin Infect Dis 2004;39:295299.
-6 Borekci S, Atahan E, Demir Yilmaz D, et al: Factors affecting the tuberculosis risk in patients receiving anti-TNF- $\alpha$ treatment. Respiration 2015;90:191-198.

$\checkmark 7$ Samandari T, Agizew TB, Nyirenda S, et al: 6-month versus 36-month isoniazid preventive treatment for tuberculosis in adults with HIV infection in Botswana: a randomised, double-blind, placebo-controlled trial. Lancet 2011;377:1588-1598. 